\title{
Comparison of Different Beamtraining Strategies from a Rate-Positioning Trade-Off Perspective
}

\author{
Jani Saloranta*, Giuseppe Destino* and Henk Wymeersch ${ }^{\dagger}$ \\ ${ }^{*}$ University of Oulu, Oulu, Finland \\ ${ }^{\dagger}$ Chalmers University of Technology, Gothenburg, Sweden \\ email: \{jani.saloranta, giuseppe.destino\}@oulu.fi, henkw@chalmers.se
}

\begin{abstract}
In next generation of mobile networks, the 5G, millimeter-wave communication is considered one of the key technologies. It allows high data rate as well as the utilization of large antennas for massive multiple-input-multiple-output (MIMO) and beamforming. However, it is mandatory that transmitter and receiver perform a training of their beams in order to gain all the benefits of a large array gain. In this paper, we study the impact of the beamtraining overhead on the data rate when an exhaustive or hierarchical strategy is used. Also, we show that the beamtraining phase can be used for positioning and, in this regard, we study the trade-off between positioning and data rate.
\end{abstract}

\section{INTRODUCTION}

Millimeter-wave communication is considered one of the key innovations of $5 \mathrm{G}$, the next generation of mobile networks. Many research studies as well as $5 \mathrm{G}$ prototypes [1] are focusing on this new technology in order to understand and verify its real performance. Due to the large available bandwidth coupled with the possibility of packing massive antenna arrays in a small form-factor, the millimeter wave (mmW) technology has the potential to enable tens of Gbps data rate as well as massive MIMO and beamforming [2]-[4].

However, one big challenge yet to be solved is the initialization of the $\mathrm{mmW}$ link in which transmitter and receiver have to align the beams to one or multiple directions in order to, for instance, maximize the signal-to-noise ratio (SNR). To address this, fast beam alignment procedures has to be devised. In [5], for instance, several beamtraining strategies have been studied and compared to each other based on the required overhead. In this paper, we specifically analyze the exhaustive and hierarchical techniques. The former is relevant for antennas capable of beamforming to fixed directions (e.g., lenses), whereas the latter is considered very efficient for reconfigurable antenna systems [1].

In contrast to previous work [6]-[8], where the objective is to investigate the performance of specific beamtraining methods, our research objectives are: $i$ ) quantify the tradeoff between the data rate and training overhead; $i i)$ determine the dependency with system parameters, such as the beamalignment periodicity (hereafter also referred to as frame length); iii) show beamtraining enables accurate positioning and $i v$ ) study the trade-off between data rate and positioning.

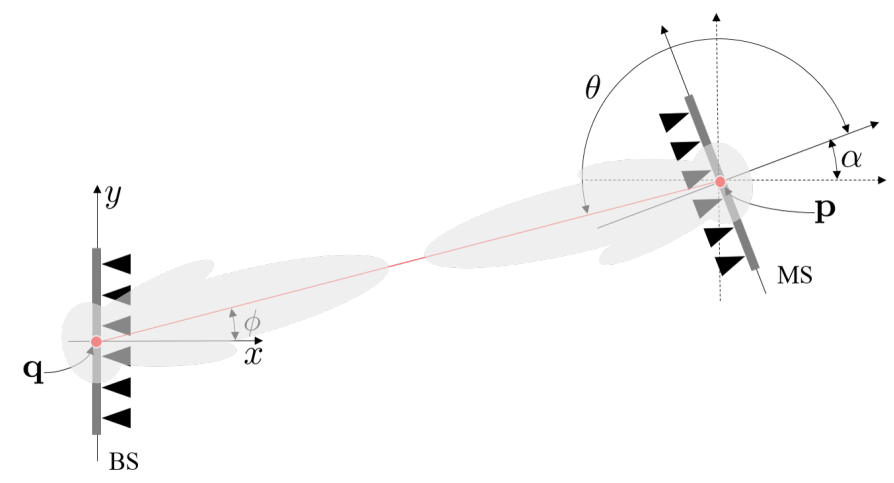

Figure 1. Geometry of the communication system including a transmitter with fixed location and orientation, and a receiver with unknown location $\mathbf{p}$ and orientation $\alpha$.

\section{MMW SINGLE-USER MIMO SYSTEM}

\section{A. Communication model}

We consider a mmW single-user MIMO wireless system with a transmitter (base station, BS) at location $^{1} \mathbf{q} \in \mathbb{R}^{2}$ and a receiver (mobile station, $\mathrm{MS}$ ) at location $\mathbf{p} \in \mathbb{R}^{2}$, equipped with an array of $N$ and $M$ antenna elements, respectively. We assume $\mathbf{q}$ known, whereas $\mathbf{p}$ is not. Also, we denote by $\alpha$ the relative 2D-rotation between the transmit and receiving antenna arrays. Figure 1 illustrates the geometry of the aforementioned communication system.

Assuming that the line-of-sight (LOS) link is the dominant path, the input-output expression for a single-user MIMO communication is

$$
y(t)=\sqrt{P_{\mathrm{tx}}} h \mathbf{w}^{\mathrm{H}} \mathbf{a}_{M}(\theta) \mathbf{a}_{N}^{\mathrm{H}}(\phi) \mathbf{f} x(t-\tau)+\mathbf{w}^{\mathrm{H}} \mathbf{n}(t),
$$

where $\mathbf{f} \in \mathbb{C}^{N}$ and $\mathbf{w} \in \mathbb{C}^{M}$ are the transmit and receive unit-norm beamforming vectors, generated from codebooks $\mathcal{F}$ and $\mathcal{W}$ with size $N_{t}^{b}$ and $N_{r}^{b}$, respectively; $\mathbf{n}(t)$ is additive white gaussian noise (AWGN) with power spectral density (PSD) $N_{0}, \tau=\|\mathbf{q}-\mathbf{p}\| / c$ is the time-delay of the LOS path for speed of light $c ; x(t)$ and $y(t)$ are the continuous timedomain transmitted and received signals of bandwidth $B$ and duration $T_{\text {sym }}$, with $1 / T_{\text {sym }} \int_{0}^{T_{\text {sym }}}|x(t)|^{2} \mathrm{~d} t=1 ; h \in \mathbb{C}$ is the

\footnotetext{
${ }^{1}$ The extension to 3-dimensions is straightforward. However, it requires the utilization of 2-dimensional antenna arrays.
} 
dominant channel coefficient; $P_{\mathrm{tx}}$ is the transmission power; $\mathbf{a}_{N}(\phi) \in \mathbb{C}^{N}$ and $\mathbf{a}_{M}(\theta) \in \mathbb{C}^{M}$ are the receive and transmit array response vectors for the angle-of-departure (AoD) $\phi$ and angle-of-arrival (AoA) $\theta$ of the dominant path. Note that $\phi$ and $\theta$ are related through $\alpha=\pi-\theta+\phi$.

We assume a uniform linear array (ULA) structure with isotropic element gain and $d=\lambda / 2$ element separation, where $\lambda$ is the carrier wavelength. In that case

$$
\left[\mathbf{a}_{M}(\theta)\right]_{m}=e^{j \frac{2 \pi d}{\lambda}(m-1) \sin (\theta)}, m \in\{1, \ldots, M\},
$$

with a similar definition for $\mathbf{a}_{N}(\phi)$. Also, we consider the case with $N=M$.

Communication occurs over frames of duration $T_{r}=$ $N_{r}^{s} T_{\text {sym }}$, of which a time $T_{t}=N_{t}^{s} T_{\text {sym }}$ is devoted to beamtraining (i.e., determining the best $\mathbf{w}$ and f), with $N_{t}^{s} \leq N_{r}^{s}$, $N_{r}^{s}, N_{t}^{s} \in \mathbb{N}_{+}$are the number of OFDM symbols in frame and number of symbols dedicated to training, respectively. Then the remainder $T_{d}=T_{r}-T_{t}$ is used for data transmission.

\section{B. Beamtraining strategies}

In this work we consider an exhaustive and an hierarchical beamtraining strategies. Also, we assume that $\mathcal{F}$ and $\mathcal{W}$ are identical, thus $N_{t}^{b}=N_{r}^{b}=N^{b}$.

1) Exhaustive beamtraining strategy: In the exhaustive beamtraining strategy, $\mathcal{F}$ and $\mathcal{W}$ are orthogonal codebooks, for instance, with $\mathbf{f}_{i}$ and $\mathbf{w}_{j}$ as the $i$-th and $j$-th column of a Discrete Fourier Transform (DFT) $N$-size matrix, respectively. Thus, the codebook size is $N^{b}=N$.

The beamtraining mechanism consists of sounding all possible transmit-receiving pairs of beams and, for each pair, measure the receiving power. After all beam-pairs have been scanned, then the receiver informs the transmitter about the beam-pair index corresponding to the maximum receiving power. Respectively, the total training time (scanning and feedback) is $T_{t}^{e}=T_{s}^{e}+T_{f}^{e}$, where

$$
\begin{aligned}
& T_{s}^{e}=T_{\text {sym }} N^{2}, \\
& T_{f}^{e}=T_{\text {sym }} .
\end{aligned}
$$

2) Hierarchical beamtraining strategy: In the hierarchical beamtraining strategy, $\mathcal{F}$ and $\mathcal{W}$ are generated based on a nested partitioning of the angular domain. In other words, we start by partitioning the whole angular domain, e.g., $[-\pi / 2, \pi / 2]$ for an ideal ULA, in $K$ bins. For each bin, we design a beamformer such that the half power beamwidth (HPBW) coincides with the upper and lower bounds of the bin. Next, each angular bin is further partitioned in $K$ bins and, a new set of beamformer is constructed. It results, that $L=\left\lceil\log _{K}(N)\right\rceil$ partitioning (also referred to as level) are performed and at for the $\ell$-level, $K^{\ell}$ beamformer vectors are defined. Then, the hierarchical beamformer codebook size is

$$
N^{b}=\sum_{\ell=1}^{\left\lceil\log _{K}(N)\right\rceil} K^{\ell}=\frac{1-K^{\left\lceil\log _{K}(N)\right\rceil+1}}{1-K}-1 .
$$

The hierarchical search strategy seeking for the link with the highest SNR is performed as divide-and-conquer algorithm.
Namely, base station (BS) and mobile station (MS) start with an exhaustive search mechanism using the $K$ beamformers of level-1. Based upon the measured receiving power, a new exhaustive search with the $K$ beamformers of level-2 is performed. This procedure iterates until beamformers of level$L$ are utilized. Respectively, the total training time (scanning and feedback) is $T_{t}^{h}=T_{s}^{h}+T_{f}^{h}$, with

$$
\begin{aligned}
& T_{s}^{h}=T_{\text {sym }} K^{2}\left(\left\lceil\log _{K}(N)\right\rceil-1\right), \\
& T_{f}^{h}=T_{\text {sym }}\left(\left\lceil\log _{K}(N)\right\rceil-1\right) .
\end{aligned}
$$

\section{Performance metrics}

We consider two performance metrics: effective data rate $R$ and position-rotation error bound (PREB).

- effective data rate: Assuming the beams selected for data transmission, after beam alignment, are $\mathbf{w}$ and $\mathbf{f}$, then

$$
R=B\left(1-\frac{T_{t}}{T_{r}}\right) \log _{2}\left(1+\frac{|h|^{2} P_{\mathrm{tx}} S(\mathbf{w}, \mathbf{f}, \theta, \phi)}{\sigma^{2}}\right),
$$

where $S(\mathbf{w}, \mathbf{f}, \theta, \phi) \triangleq\left|\mathbf{w}^{H} \mathbf{a}_{M}(\theta)\right|^{2}\left|\mathbf{f}^{H} \mathbf{a}_{N}(\phi)\right|^{2}, \sigma^{2}=$ $N_{0} B$ is the noise power over the signal bandwidth. Note that for a fixed $T_{t}$, the rate is maximized when $\mathbf{f}=1 / \sqrt{N} \mathbf{a}_{N}(\phi)$ and $\mathbf{w}=1 / \sqrt{M} \mathbf{a}_{M}(\theta)$.

- PREB: Assuming a total of $K$ transmit/receive beam combinations $\left(\mathbf{f}_{k}, \mathbf{w}_{k}\right)$ are used, we can compute the Fisher Information Matrix (FIM) associated with $[\mathbf{p}, \alpha]$. The PREB is the inverse of this FIM, and provides a lower bounds, in the positive semi-definite sense on the achievable estimation accuracy of $[\mathbf{p}, \alpha]$. The mathematical definition of PREB will be provided in Section III-C.

Generally, increasing the number of training beams will improve the PREB, but also lead to an increase in $T_{t}$. However, we shall see that based upon the searching strategy, the increase of $T_{t}$ does not always compromise the increase of data rate. Also that tension between rate and PREB does not always subsist. Our goal is to understand and quantify this trade-off.

\section{RATE AND PREB}

\section{A. Effective data rate for exhaustive search}

Consider the exhaustive search strategy described in Section II-B1. The effective data rate is given by

$$
R=B\left(1-\frac{\left(N^{2}+1\right)}{N_{r}^{s}}\right) \log _{2}\left(1+\frac{|h|^{2} P_{\mathrm{tx}} \mathrm{S}\left(\mathbf{w}^{*}, \mathbf{f}^{*}, \theta, \phi\right)}{\sigma^{2}}\right),
$$

in which the optimal beams are those that maximize the SNR:

$$
\left[\mathbf{w}^{*}, \mathbf{f}^{*}\right]=\arg \max _{\substack{\mathbf{w} \in \mathcal{W} \\ \mathbf{f} \in \mathcal{F}}} \frac{|h|^{2} P_{\mathrm{tx}} \mathrm{S}(\mathbf{w}, \mathbf{f}, \theta, \phi)}{\sigma^{2}} .
$$

Under the condition that beamformers are perfectly aligned with the $\theta$ and $\phi$, then $\mathrm{S}(\mathbf{w}, \mathbf{f}, \theta, \phi)=N^{2}$. It follows that

$$
\begin{aligned}
R & =B\left(1-\frac{\left(N^{2}+1\right)}{N_{r}^{s}}\right) \log _{2}\left(1+\mathrm{SNR}_{0} N^{2}\right), \\
& \approx 2 B\left(1-\frac{N^{2}}{N_{r}^{s}}\right) \log _{2}\left(\mathrm{SNR}_{0} N\right)
\end{aligned}
$$


where $\mathrm{SNR}_{0} \triangleq|h|^{2} P_{\mathrm{tx}} / \sigma^{2}$ and (12) holds for $N \gg 1$.

By computing the derivative of (11) with respect to $x=N^{2}$

$$
\frac{\partial R\left(x ; S N R_{0}\right)}{\partial x}=0,
$$

then the maximum rate is achieved when $x$ satisfies

$$
x=N_{r}^{s}-1-\frac{\left(1+\mathrm{SNR}_{0} x\right)}{\mathrm{SNR}_{0}} \ln \left(1+\mathrm{SNR}_{0} x\right) .
$$

Subsequently, when $T_{t}^{e}=\left\lceil x_{\mathrm{opt}}\right\rceil T_{\text {sym }}$, where $x_{\mathrm{opt}}$ is the solution to the above equation.

\section{B. Effective data rate for hierarchical search}

Consider the hierarchical search strategy described in II-B2. The effective rate is given by

$$
R=\left(1-\frac{\left(K^{2}+1\right)\left\lceil\log _{K}(N)\right\rceil}{N_{r}^{s}}\right) \log _{2}\left(1+\frac{|h|^{2} P_{\mathrm{tx}} \mathrm{S}\left(\mathbf{w}^{*}, \mathbf{f}^{*}, \theta, \phi\right)}{\sigma^{2}}\right),
$$

with $\mathbf{w}^{*}, \mathbf{f}^{*}$ satisfying equation (10).

Likewise the study of the exhaustive search, we assume that beams obtained with $\mathbf{w}^{*}$ and $\mathbf{f}^{*}$ are perfectly aligned with the AoA and AoD. Hence, the maximum rate can be computed as

$$
\begin{aligned}
R & =B\left(1-\frac{\left(K^{2}+1\right)\left\lceil\log _{K}(N)\right\rceil}{N_{r}^{s}}\right) \log _{2}\left(1+\mathrm{SNR}_{0} N^{2}\right) \\
& \approx 2 B\left(1-\frac{\left(K^{2}+1\right)\left\lceil\log _{K}(N)\right\rceil}{N_{r}^{s}}\right) \log _{2}\left(\mathrm{SNR}_{0} N\right)
\end{aligned}
$$

where the approximation holds for $N \gg 1$.

Based on this result, the following remarks are in order: $i$ ) $T_{t}^{h}(N) \in \mathcal{O}\left(\log _{K}(N)\right)$, ii) $T_{t}^{h}(N) \in o\left(T_{t}^{e}(N)\right)$, and $\left.i i i\right)$ $T_{t}^{h}(N)<\left(\log _{2}\left(\mathrm{SNR}_{0} N\right)\right)$ for a sufficiently large $\mathrm{SNR}_{0}$. In words, $i$ ) indicates that with an hierarchical search, the beamtraining overhead increase with $N$, but sublinearly; $i i$ ) shows that the hierarchical search is much more over timeefficient than the exhaustive search and, more importantly, $i i i$ ) demonstrates that the array gain is the dominant factor for a rate increase. Thus by increasing $N$, the rate grows despite of a longer training time.

For the sake of completeness, Figure 2 shows the comparison of different strategies in terms of the training overhead (number of slots) as a function of the number of antenna elements. More specifically, for the hierarchical search we consider a binary $(K=2)$ and ternary $(K=3)$ partitioning.

\section{PREB from Fisher information}

1) PREB analysis: Introducing $\boldsymbol{\xi} \triangleq\left[\mathbf{p}^{\mathrm{T}}, \alpha, h\right]^{\mathrm{T}}$ with an associated FIM $\mathbf{J}_{\xi}$, obtained from aggregating information during the exhaustive beam alignment strategy, the PREB comprised two components: the position error bound

$$
\mathrm{PEB}=\sqrt{\operatorname{trace}\left(\left[\mathbf{J}_{\xi}^{-1}\right]_{1: 2,1: 2}\right)},
$$

expressed in meters, and the rotation error bound

$$
\mathrm{REB}=\sqrt{\left[\mathbf{J}_{\xi}^{-1}\right]_{3,3}}
$$

expressed in radians (or degrees, after conversion).

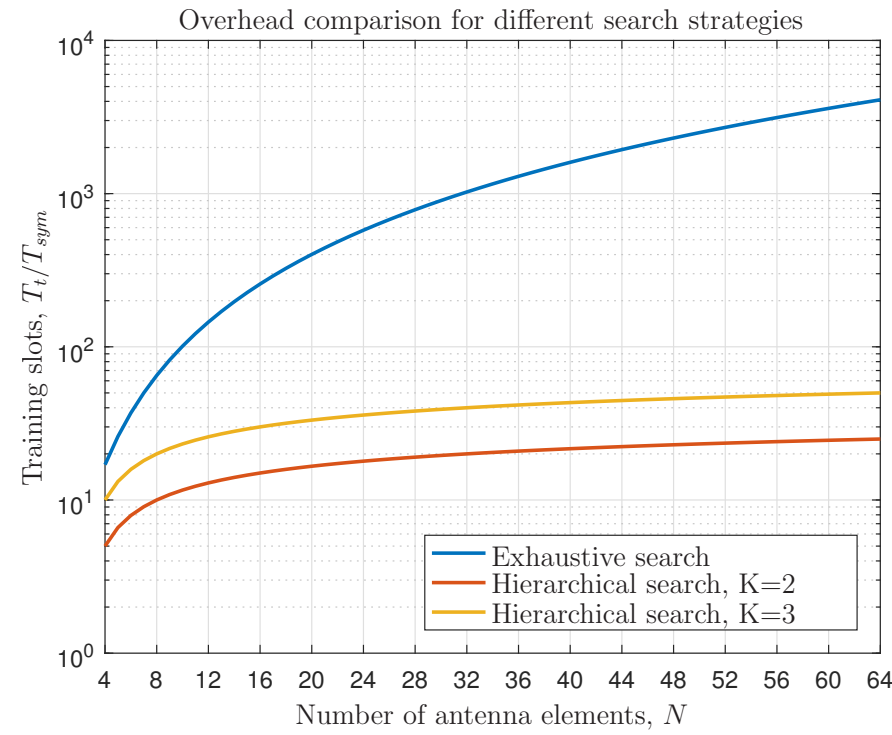

Figure 2. Comparison of different training strategies in terms of training slots.

Due to the additive nature of Fisher information,

$$
\mathbf{J}_{\xi}=\sum_{\substack{\mathbf{w} \in \mathcal{W} \\ \mathbf{f} \in \mathcal{F}}} \mathbf{J}_{\boldsymbol{\xi}}(\mathbf{f}, \mathbf{w})
$$

where the FIM associated with a single beam pair. Hence, we only need to determine an expression $\mathbf{J}_{\boldsymbol{\xi}}(\mathbf{f}, \mathbf{w})$.

2) FIM for a single beam: Given (f, w), the $\operatorname{FIM~} \mathbf{J}_{\boldsymbol{\xi}}(\mathbf{f}, \mathbf{w})$ will be derived, from the observation model (1) as follows. We express the deterministic part of the received signal $y(t)$, utilized by the receiver for position-rotation estimation, by $y(t) \triangleq \sqrt{P_{\mathrm{tx}}} \mathbf{w}^{\mathrm{H}} h \mathbf{a}_{M}(\theta) \mathbf{a}_{N}^{\mathrm{H}}(\phi) \mathbf{f} x(t-\tau)$. Also, we derive the geometric relationship between the vector $\boldsymbol{\eta} \triangleq[h, \theta, \phi, \tau]^{\mathrm{T}}$ including the channel parameters with the unknown position $\mathbf{p}$ and rotation $\alpha$ as

$$
\left[\begin{array}{c}
p^{x} \\
p^{y} \\
\alpha
\end{array}\right]=\left[\begin{array}{c}
q^{x}+c \tau \cos (\phi) \\
q^{y}+c \tau \sin (\phi) \\
\pi+\phi-\theta
\end{array}\right],
$$

where $q^{x}$ and $q^{y}$ are the $x$ and $y$ coordinates of the known transmitter location, respectively.

The PREB can be computed from the inverse of the FIM $\mathbf{J}_{\boldsymbol{\xi}}(\mathbf{f}, \mathbf{w})$ given by

$$
\mathbf{J}_{\boldsymbol{\xi}}(\mathbf{f}, \mathbf{w})=\mathbf{T} \mathbf{J}_{\boldsymbol{\eta}}(\mathbf{f}, \mathbf{w}) \mathbf{T}^{\mathrm{T}}
$$

where $\boldsymbol{\xi} \triangleq\left[\mathbf{p}^{\mathrm{T}}, \alpha, h\right]^{\mathrm{T}}$ and $\mathbf{T} \in \mathbb{R}^{5 \times 5}$ is obtained as

$$
\mathbf{T}=\left[\begin{array}{cc}
\mathbf{0} & \boldsymbol{\Upsilon} \\
\mathbf{I}_{2} & \mathbf{0}
\end{array}\right]
$$

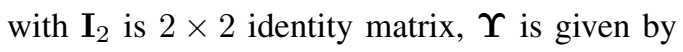

$$
\mathbf{\Upsilon}=\frac{1}{c}\left[\begin{array}{ccc}
-\sin (\phi) / \tau & -\sin (\phi) / \tau & \cos (\phi) \\
\cos (\phi) / \tau & \cos (\phi) / \tau & \sin (\phi) \\
-c & 0 & 0
\end{array}\right],
$$

and $\mathbf{J}_{\boldsymbol{\eta}}(\mathbf{f}, \mathbf{w}) \in \mathbb{R}^{5 \times 5}$ is a symmetric matrix defining the FIM for the estimation of the channel parameters $\boldsymbol{\eta}$ from beam pairs $(\mathbf{f}, \mathbf{w})$. Detail expression of $\mathbf{J}_{\boldsymbol{\eta}}$ is given in [9], [10]. 


\section{Simulation RESUlts}

In this section, we study the impact of the different search strategies and the codebook size, both on the effective data rate and position-rotation bound as well as the trade-off between these two metrics.

\section{A. Simulation setup}

We focus on an idealized MIMO-orthogonal frequencydivision multiplexing (OFDM) communication system with 2048 subcarriers, inter-carrier spacing $\Delta_{f}=75 \mathrm{kHz}$, transmission power $P_{\mathrm{tx}}=30 \mathrm{dBm}$, number of antenna elements on BS and MS side same $N=M$, and carrier frequency 28 GHz. Then one beam during beam alignment corresponds to one OFDM symbol, so $T_{\text {sym }}=13 \mu \mathrm{s}$ and $E_{s}=P_{\mathrm{tx}} T_{\text {sym }}$, and a radio frame with $N_{r}^{s}=\left\lceil T_{r} / T_{\text {sym }} \eta\right\rceil$ OFDM symbols, where $\eta$ is the OFDM time-efficiency, e.g., $\eta=0.93$.

The MS location is at a fixed distance of $100 \mathrm{~m}$ to the BS (corresponding to $\mathrm{SNR}_{0} \approx 20 \mathrm{~dB}$ ) and $\alpha=0^{\circ}$ (no rotation). We study the cases $\phi=0^{\circ}$ and $\phi=20^{\circ}$, allowing us to analyze the effect of beam misalignment.

\section{B. Trade-off between rate and training overhead}

The first result, illustrated in Figure 3, shows the achievable rate as a function of the frame length $T_{r}$ with the exhaustive and hierarchical training strategies.

Let us focus on the exhaustive search (blue lines) and compare the case between $N=8$ (solid line) and $N=32$ (dashed line). It can be noticed that for $T_{r} \leq 55 \mathrm{~ms}$ the highest rate is achieved with $N=8$. This is due to high rate-loss caused by the training of $N=32$ beams per transmit and receiver. In contrast, with the hierarchical search the highest rate is achieved with $N=32$ despite of the frame length.

The next result, shown in Figure 4, tackles the problem of beam misalignment and, more specifically, we evaluate the effective rate as a function of the training time (obtained by varying the number of antennas) by changing the location of the receiver. Also in this plot, it can be noticed the exhaustive search strategy has an optimum training time whereas the rate achieved with hierarchical search grows despite the increase of $T_{t}$. More importantly, the rate obtained with the exhaustive search is also function of the $\phi$ and oscillates based upon the choice of $T_{t}$ as, the latter depends on $N$, thus on the beamformers in the codebook. This phenomena is not visible with the hierarchical search as the codebook is denser in the angular domain.

\section{Positioning and data rate trade-off}

In this Section we study the trade-off between positioning performance and effective data rate. As mentioned in Section III-C, positioning information can increase with the number of sounded beam pairs. If the training period is used also for positioning, then the longer the training, the lower the positioning error. Therefore, quantifying the achievable positioning error and assess which strategy can provide the best positioning performance are our first objectives.

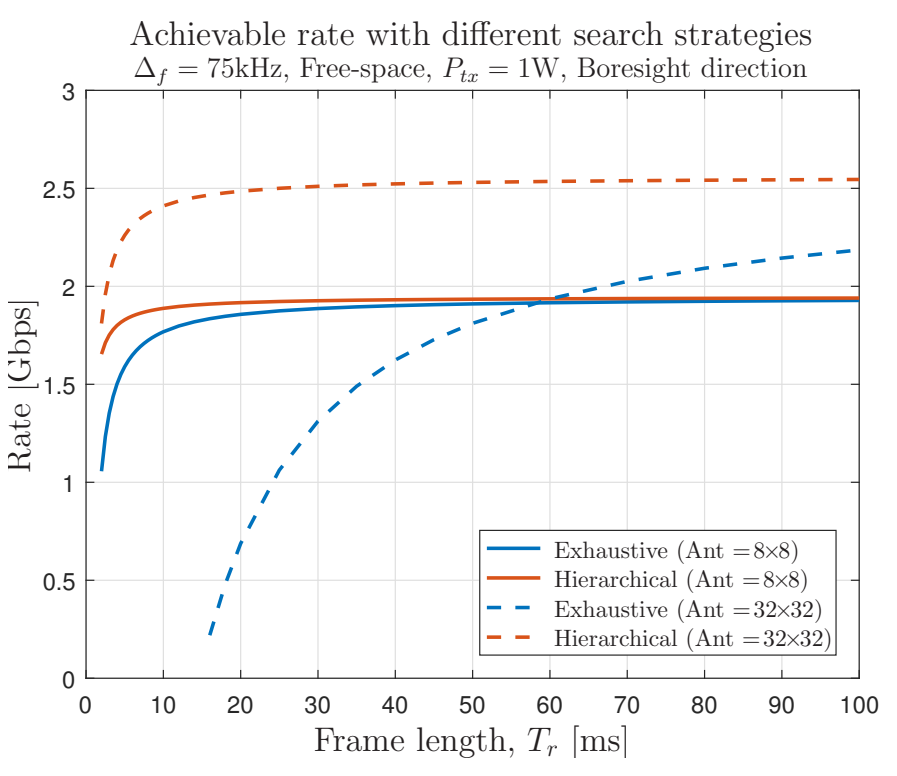

Figure 3. Comparison on the achievable rate with perfect beam-channel alignment. Rate as a function of the frame length and varying the number of antennas. Trade-off between training overhead and $\phi$

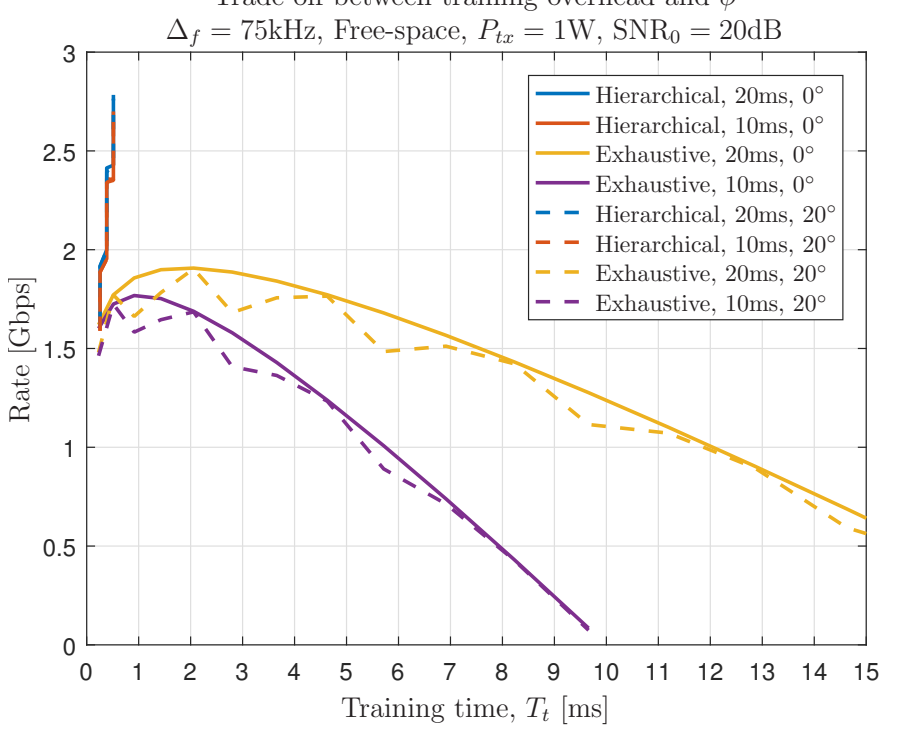

Figure 4. Comparison on the achievable rate with perfect beam-channel alignment. Rate as function of the frame length $\left(T_{r}=\{10 \mathrm{~ms}, 20 \mathrm{~ms}\}\right)$ and varying the location $\left(\phi=\left\{0^{\circ}, 20^{\circ}\right\}\right)$.

Figure 5 shows the progress of the position error bound (PEB) as a function of the number of training slots. More specifically, the result refers to a set-up with $N=M=32$ antennas, frame length $T_{r}=\{10 \mathrm{~ms}, 20 \mathrm{~ms}\}$ and $\phi=\left\{0^{\circ}, 20^{\circ}\right\}$.

Our first observation is that better positioning performance can be achieved with the hierarchical search despite of the small number of training slots. In fact, by progressively aligning the beams, more information is captured. Also, due to a sufficient density of beamformers, the performance is invariant respect to $\phi$. In contrast, the exhaustive search yields a larger location error, that ultimately remains invariant with $\phi$. 


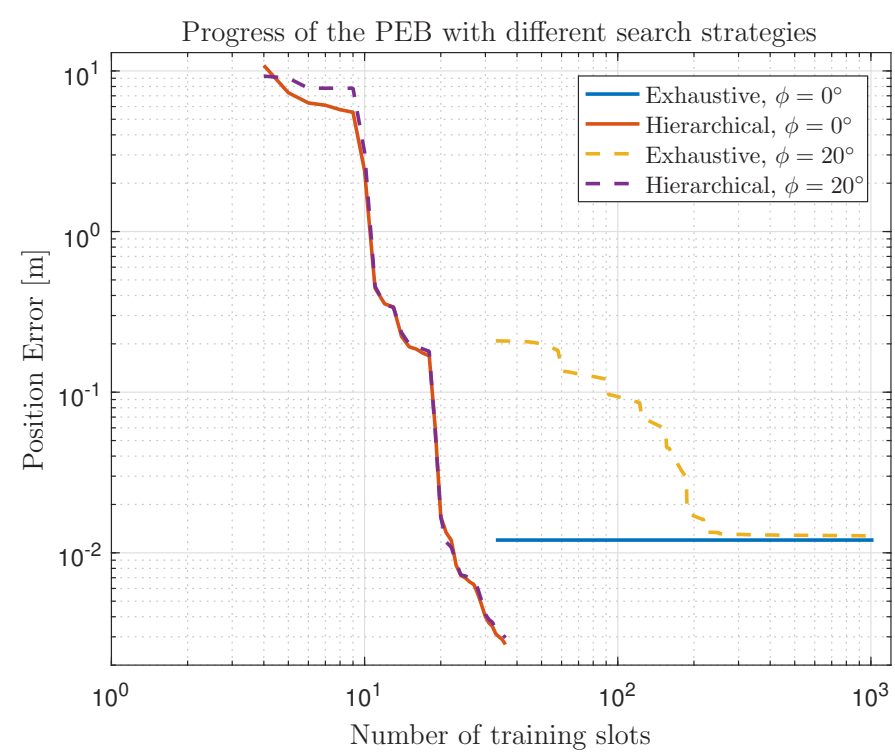

Figure 5. Position error bound as function of number of slots used in Exhaustive vs Hierarchical search strategies. $\mathrm{SNR}=20 \mathrm{~dB}$ in a Boresight $\left(\phi=0^{\circ}\right)$ and misalignment $\left(\phi=20^{\circ}\right)$ scenarios.

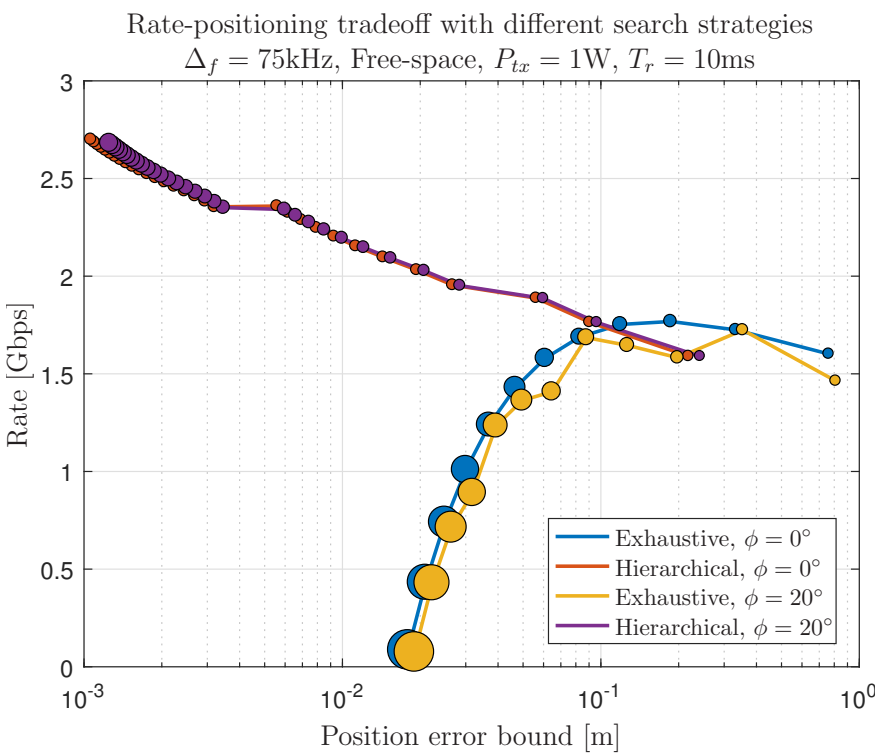

Figure 6. Comparison of the relationship between rate and PEB. A trade-off is noticeable with the exhaustive search. Marker size is reflecting to the

However, the progress of the PEB depends on the location and on the sweeping order of the beams. In the proposed simulation, the boresight direction is the first to be sounded by both transmitter and receiver. Therefore, with $\phi=0^{\circ}$, the lowest PEB is reached already after the first sweep $^{2}$. Also, there is no increase of information (decrease of error), as all transmitter and receiver beams, but the boresight, have a null at $\phi=0^{\circ}$. On the other hand, with $\phi=20^{\circ}$, none of the codebook beamformers have neither maximum gain or null at $\phi$. Thus, a continuous descend of the error occurs.

Finally, Figure 6 shows the relationship between effective rate and positioning error with different searching strategies.

\footnotetext{
${ }^{2} \mathrm{~A}$ necessary condition for the inversion of the position FIM is that both transmitter and receiver sounds the channel with at least two beams [9].
}

Also, the results are drawn with $\phi=0^{\circ}, \phi=20^{\circ}$ and the size of each marker is proportional to the overhead. With an exhaustive search a trade-off must be met to either increase the rate or decrease the position error. The reason of this tradeoff is the time-inefficiency of the training. In contrast, the relationship between PEB and rate with the hierarchical training does not show any trade-off. Indeed, increasing the rate will also increase the positioning performance. Furthermore, the dependency with $\phi$ is only noticeable with the exhaustive search.

\section{CONCLUSion}

In this paper we investigated different beamtraining strategies to look into the trade-off between achievable data rate and training overhead as well as with positioning. We show that in exhaustive search strategy a trade-off must be met to either increase the achievable data rate or decrease the position error, in contrast hierarchical beamtraining method does not show any trade-off between PEB and data rate. Particularly, we confirm that beamtraining enables accurate positioning.

\section{ACKNOWLEDGMENTS}

The research leading to these results has received funding from the Academy of Finland, Fundamental of Simultaneous Localization and Communications (FALCON) as well as the European Union H2020 5GPPP under grant no. 723247 and supported by the Institute for Information \& communications Technology Promotion (IITP) grant funded by the Korea government (MSIP) (No.B0115-16-0001, 5GCHAMPION).

\section{REFERENCES}

[1] M. Mueck et al., "5G CHAMPION - Rolling out 5G in 2018," in Proc. IEEE Global Commun. Conf. Workshops, Dec. 2016, pp. 1-6.

[2] Z. Pi and F. Khan, "An introduction to millimeter-wave mobile broadband systems," IEEE Commun. Mag., vol. 49, no. 6, pp. 101-107, Jun. 2011.

[3] P. Wang, Y. Li, L. Song, and B. Vucetic, "Multi-gigabit millimeter wave wireless communications for $5 \mathrm{~g}$ : from fixed access to cellular networks," IEEE Commun. Mag., vol. 53, no. 1, pp. 168-178, Jan. 2015.

[4] R. W. Heath, N. González-Prelcic, S. Rangan, W. Roh, and A. M. Sayeed, "An Overview of Signal Processing Techniques for Millimeter Wave MIMO Systems," IEEE J. Sel. Topics Signal Process., vol. 10, no. 3, pp. 436-453, Apr. 2016.

[5] S. Kutty and D. Sen, "Beamforming for Millimeter Wave Communications: An Inclusive Survey," IEEE Commun. Surveys Tuts., vol. 18, no. 2, pp. 949-973, 2016.

[6] Z. Xiao, T. He, P. Xia, and X. G. Xia, "Hierarchical codebook design for beamforming training in millimeter-wave communication," IEEE Trans. Wireless Commun., vol. 15, no. 5, pp. 3380-3392, May 2016.

[7] C. Liu, M. Li, I. B. Collings, S. V. Hanly, and P. Whiting, "Design and Analysis of Transmit Beamforming for Millimeter Wave Base Station Discovery," IEEE Trans. Wireless Commun., vol. 16, no. 2, pp. 797-811, Feb. 2017.

[8] B. W. Ku, D. G. Han, and Y. S. Cho, "Efficient Beam-Training Technique for Millimeter-Wave Cellular Communications," ETRI Journal, vol. 38, no. 1, pp. 81-89, 2016.

[9] A. Shahmansoori, G. E. Garcia, G. Destino, G. Seco-Granados, and H. Wymeersch, "5G Position and Orientation Estimation through Millimeter Wave MIMO," in Proc. IEEE Global Commun. Conf. Workshops, Dec. 2015, pp. 1-6.

[10] G. Destino and H. Wymeersch, "On the Trade-off Between Positioning and Data-Rate for mm-Wave Communications," in Proc. IEEE Int. Conf. Commun. Workshop, May 2017. 